\title{
Georgia Santangelo dir., Les maîtres de l'eau, d'Archimède à la machine de Marly
}

Musée-promenade, Marly-le-roi / Louveciennes, Éditions Artlys, 2006, 176 pages.

\section{Michèle Virol}

\section{(Q) OpenEdition}

\section{Journals}

Édition électronique

URL : http://journals.openedition.org/dht/596

DOI : $10.4000 /$ dht.596

ISSN : 1775-4194

Éditeur :

Centre d'histoire des techniques et de l'environnement du Cnam (CDHTE-Cnam), Société des élèves du CDHTE-Cnam

\section{Édition imprimée}

Date de publication : 31 mars 2009

Pagination : 236-238

ISBN : $978-2-95-30779-3-3$

ISSN : 0417-8726

Référence électronique

Michèle Virol, « Georgia Santangelo dir., Les maîtres de l'eau, d'Archimède à la machine de Marly », Documents pour I'histoire des techniques [En ligne], 17| $7^{\text {er }}$ semestre 2009, mis en ligne le 27 septembre 2010, consulté le 24 septembre 2020. URL : http://journals.openedition.org/dht/596 ; DOI : https:// doi.org/10.4000/dht.596

Ce document a été généré automatiquement le 24 septembre 2020.

(c) Tous droits réservés 


\section{Georgia Santangelo dir., Les maîtres de l'eau, d'Archimède à la machine de Marly}

Musée-promenade, Marly-le-roi / Louveciennes, Éditions Artlys, 2006, 176 pages.

\section{Michèle Virol}

\section{RÉFÉRENCE}

Georgia Santangelo dir., Les maîtres de l'eau, d'Archimède à la machine de Marly, Muséepromenade, Marly-le-roi / Louveciennes, Éditions Artlys, 2006, 176 pages.

1 La publication de ce bel ouvrage magnifiquement illustré de documents d'archives accompagnait une exposition qui se déroula au Musée-Promenade de Marly-le-roi/ Louveciennes du 4 mars au 30 juillet 2006. La machine de Marly, conçue pour satisfaire le souhait de Louis XIV de "faire de l'eau de ses jardins le miroir de sa puissance », est au cœur des neuf contributions rassemblées dans ce livre mais le lecteur est aussi invité à parcourir les siècles en embrassant l'historique des machines à eau depuis l'antiquité (Robert Halleux) jusqu'aux techniques de la «nouvelle machine pour Marly» (Bruno Jacomy).

Pour évaluer cette spectaculaire réalisation, Robert Halleux rappelle qu'au XVII ${ }^{e}$ siècle les seuls moteurs disponibles " pour mouvoir toute espèce de machines et notamment celles pour élever l'eau" sont la cage d'écureuil, le manège à chevaux, la roue hydraulique et le moulin à vent. Le mouvement circulaire continu qu'ils produisent peut être transformé par un des trois mécanismes: engrenage à angle droit, arbre à cames (connus dès l'Antiquité) ou le système bielle-manivelle reproduit dans les cahiers d'ingénieurs du XV siècle. A la Renaissance, la combinaison de ces mécanismes simples a permis la floraison de machines nouvelles reproduites dans les théâtres de machines 
et a trouvé une large application dans l'hydraulique minière comme l'atteste le De re metallica (1556) du médecin saxon Georg Bauer dit Agricola.

3 La contribution d'André Guillerme ("L'aquosité au XVII siècle») souligne l'intérêt esthétique et philosophique pour l'eau durant le grand siècle. Élément indispensable des fêtes, des promenades et du bien-être, l'eau domestiquée se trouve souvent magnifiée mais sert également, de façon plus guerrière, aux systèmes défensifs des places-fortes. Elle est au centre de débats scientifiques entre partisans du cycle souterrain qui affirment qu'elle provient du centre de la terre et défenseurs du cycle atmosphérique convaincus par les démonstrations de l'astronome Halley.

La nécessité de « conduire » les eaux s'impose pour satisfaire une demande de plus en plus pressante et les techniques d'exhaure (évacuation de l'eau) des mines servent l'hydraulique somptuaire. La machine de Marly est le résultat de ce transfert de technologie. Ses constructeurs originaires de Liège, Arnold de Ville et le charpentier Rennequin Sualem ont transposé, en changeant d'échelle, les connaissances maîtrisées dès le $\mathrm{XVI}^{\mathrm{e}}$ siècle dans la principauté. L'industrie wallonne reposant sur l'extraction minière et la production de fonte par réduction indirecte, la transformation de l'énergie hydraulique en travail par des machines en bois ou la résolution des nappes aquifères dans les extractions minières ont donné une grande impulsion à l'invention et à l'utilisation de machines d'exhaure. Les mécanismes utilisés à Marly y sont déjà employés : roues à aube, manivelles, pompes, mécanismes de transmission. De plus, l'hydraulique domestique (telle l'adduction d'eau réalisée à Liège par "l'ingénieur " Jean Roland) et l'hydraulique somptuaire (les jets d'eau et fontaines de jardins des résidences des archiducs à Mariemont et Tervuren et surtout la machine de Modave, près de Huy, qui élève l'eau d'une quarantaine de mètres dans le château de JeanGaspard de Marchin en 1675 et 1676) attestent du transfert technique déjà réalisé dans la principauté. Les constructeurs de la machine de Marly, Rennequin Sualem (1645-1708), fils de charpentier de machines, son frère et leur beau-frère Gilles Lambotte sont issus de cette culture technique.

5 Le besoin en eau des jardins de Versailles que n'ont pas satisfait les vastes entreprises d'adduction (l'étang de Clagny, la pompe à chevaux de Denis Jolly, le détournement de la Bièvre, le réseau de plus de 200 kilomètres de rigoles et d'aqueducs, le stockage dans les étangs de plus de 8 millions de mètres cubes) est à l'origine en 1678 de l'acceptation de la proposition du chevalier Arnold de Ville (1653-1722) d'une machine à refouler l'eau de la Seine sur une pente de plus de 150 mètres. Ce fils de maitre des forges et bourgmestre de Huy qui a été initié aux mathématiques par les Jésuites fait la promotion des compétences industrielles liégeoises. Les travaux débutent en 1680 et s'achèvent en 1685. La machine propose la multiplication en batterie de chaque élément constitutif (roue, pompe, mécanisme de transmission) entraînant des contraintes mécaniques qui vont finalement être des obstacles à son bon fonctionnement. L'eau est montée en trois temps et à chaque étape, elle est stockée avec les eaux drainées du coteau dans un réservoir ou puisard, l'étanchéité des jointures des multiples tuyaux étant assurée par des pièces de cuir et des joints de plomb. Raphaël Morera insiste sur la démesure plus que sur la nouveauté de la machine avec ses 259 pompes, ses 14 roues de 36 pieds $(11,6 \mathrm{~m})$ de diamètre réparties sur trois rangées. Les travaux de maçonnerie préparatoires et indispensables ont absorbé les deux tiers des dépenses (près de deux millions de livres), le coût du métal ne s'élevant qu'à 750000 livres soit moins du quart. A partir de 1689, les dépenses sont 
essentiellement des dépenses d'entretien, main d'œuvre et remplacement des pièces usées. La construction a nécessité 1800 hommes et une soixantaine pour l'entretien. Les pannes répétées signalent que la machine n’a jamais été au maximum de ses capacités, et fonctionnant nuit et jour, elle a exigé de la part des ouvriers un travail continu. Véritable entreprise, elle a sa vie propre, ses règlements et ses dérèglements (vols de fournitures, actes de vandalisme, absentéisme, etc.) soulignés par Georgia Santangelo qui met en évidence la force symbolique de cette réalisation sous le titre éloquent de "La machine : rêves et cauchemars ».

Bruno Bentz dévoile grâce aux fouilles archéologiques menées sur le site que les jets d'eau de Marly ont pu fonctionner grâce à un réseau complètement autonome dès 1683, avant même l'arrivée des eaux de la Seine qui modifia peu ce premier réseau. La machine a cependant permis une meilleure alimentation, surtout sans relâche, des aqueducs, réservoirs et galeries, apportant aux jardins ses cascades et ses nombreux jets d'eau dont le plus haut est à plus de 30 mètres, et ce presque jusqu'à la fin du règne de Louis XIV.

7 La machine est emblématique de la volonté d'affirmation de la puissance royale. Quand l'eau est rare dans les villes, le monarque la "gaspille » dans les jets d'eau pour mettre en scène son pouvoir avec plus d'ostentation. Elle est devenue un monument, mais aussi un monstre, un objet de rêve et de jalousies. La machine a une histoire, attachée certes aux fastes du Roi-Soleil, mais même après son abandon temporaire de 1722 à 1739, en raison du coût de son entretien et de la désaffection de Versailles sous la Régence, elle ne va cesser d'exciter les ingénieurs et inventeurs qui, tantôt proposent son remplacement, tantôt veulent la perfectionner. Wilfrid Eon met en évidence le rôle entre 1745 et 1773, du marquis de Marigny, directeur général des Bâtiments du roi, qui prête une attention particulière aux projets qui proposent de sortir la machine de l'état de délabrement dans lequel elle se trouve, accordant à ceux de l'académicien mathématicien Antoine Deparcieux et surtout de Van Bockstaël, ingénieur du duc de Lorraine et roi de Pologne Stanislas Leszczynski, beaucoup d'intérêt. Finalement, alors que les travaux ont commencé, il renonce à la proposition qui techniquement l'avait séduit en raison de son coût pourtant volontairement sous-estimé par Van Bockstaël. La monarchie ne peut investir les sommes colossales demandées et le personnel attaché à la machine tente d'empêcher toute modification. Ce phénomène est récurrent et lorsque la machine relève de la compétence scientifique et technique de l'Académie des sciences qui lance des concours pour favoriser la formulation de projets, les académiciens vont aussi se heurter à ces difficultés, selon Sylvie Provost, notamment Charles-Augustin Coulomb et Jacques-Constantin Périer qui s'intéressent à la machine de 1784 à 1810. Les Anglais Watt et Boulton, sollicités en 1786 pour apporter leurs solutions, déclinent l'offre car ils comprennent que les enjeux ne sont pas uniquement techniques mais aussi financiers et politiques.

8 Les savants se heurtent aussi à la résolution de questions techniques relevant de la mécanique des fluides pour remplacer les pompes de la machine, les connaissances d'hydrodynamique étant insuffisantes. Les solutions concrètes n'interviendront qu'après 1800 et seront partiellement mises en œuvre dans la première moitié du XIX ${ }^{e}$ siècle. Après avoir écarté le projet de l'ingénieur bavarois Joseph von Baader, le projet de pompe à feu associé à une installation hydraulique réduite à deux roues de Cécile et Martin est mis en service en 1823 et fonctionnera jusqu'en 1856. Puis la machine à vapeur est abandonnée pour une nouvelle machine hydraulique, celle de Dufrayer mise 
en service en 1858 et restée en usage jusqu'en 1963. Pour Bruno Jacomy, ces tentatives avortées s'expliquent par le fait que la machine de Marly est un véritable système qui met en jeu plusieurs fonctions (captage de l'eau, énergie motrice, pompage et amenée de l'eau jusqu'à l'aqueduc) qu'il faut chacune améliorer. Les progrès techniques effectifs au milieu du XIX ${ }^{\mathrm{e}}$ siècle, notamment pour les moteurs hydrauliques rendent opérationnelle et plus économique le seul projet vraiment abouti, la machine de Dufrayer.

9 Le lecteur pourrait regretter, après avoir suivi la genèse et les péripéties de cette machine au fil des articles et des superbes reproductions de documents d'archives, textes, dessins et peintures qu'une réflexion sur l'enjeu patrimonial de certaines machines ne vienne clore l'ouvrage. Les répétitions inhérentes au partage des sujets parfois très proches ont accentué une approche de la machine comme objet doté d'une vie propre liée à ses fonctions, son entretien, son coût, sa charge symbolique générant des résistances parfois insurmontables lorsqu'il fallait la transformer ou la remplacer. La machine de Marly appartient au registre d'objet que l'histoire s'est approprié, lui donnant une charge emblématique très forte qu'il est nécessaire de décrypter.

10 Ce livre magnifiquement présenté contribue par ses articles pertinents et savants à dévoiler ces enjeux.

\section{AUTEURS}

\section{MICHÈLE VIROL}

Centre Roland Mousnier-UMR 8596 